\title{
IMPROVING THE PRODUCTIVITY OF BAHEIJ CHICKENS THROUGH BOTH OF BACKCROSSING AND SELECTION FOR BODY WEIGHT AT 8 WEEKS OF AGE C. GROWTH TRAITS, BODY CONFORMATION, CHICK VIABILIT, AND CARCASS TRAITS
}

\author{
Yousria K. M. Afifi*; Osama M. Aly; Nema A. Mosaad; \\ Nazla Y. Abou El-Ella; and Magda M. Balat
}

Anim. Prod. Res. Inst., Agric. Res. Center, Ministry of Agric., Dokki, Egypt

*Corresponding author: Yousria K. M. Afifi; E-mail:yousriaafifi2006@yahoo.com

Received:11/10/2016 Accepted:05/02/2017

\begin{abstract}
Comparison study was carried out among three genotypes of Baheij breed, Baheij selected breed for increasing body weight at 8 weeks of age, Baheij upgraded groups through three generations and control group. Results were as follows:

1 - Chicks of the $3^{\text {rd }}$ generation were the heaviest $(\mathrm{p}<0.001)$ compared to those of the $1^{\text {st }}$ and $2^{\text {nd }}$ one at the different studied ages. Also, means of body weight of the upgraded group was the highest $(p<0.001)$ compared to the other two groups and the selected group surpassed $(p<0.001)$ the control one at all ages studied.

2- The three studied factors affected significantly growth rate percentage (GR \%) where birds of the $3^{\text {rd }}$ generation had the highest value of GR\% through most of the studied intervals. The upgraded group had the highest mean at 12-16 wks of age where, the selected group grew faster at 8-12 wks of age. Both of the upgraded and the selected groups had significantly the highest values compared with the control one at $0-12,12-20$ and at $0-16$ wks of age, while both of the upgraded and control groups surpassed $(\mathrm{p}<0.001)$ the selected one at $12-16$ wks of age.

3- Body weight (BW), shank length (SL), keel length (KL) and breast width had significantly $(\mathrm{p}<0.01)$ higher averages at 12 wks. of age compared to those at 8 wks of age. Also, the selected group had significantly the highest BW while, the upgraded group had the highest average of SL. Moreover, males were heavier $(\mathrm{p}<0.01)$ than females. 4 - The birds of the $2^{\text {nd }}$ generation had the best $(p<0.01)$ chick viability at $0-4$ wks of age, while the values of both of the $1^{\text {st }}$ and $3^{\text {rd }}$ generations were nearly similar and higher than that of the $2^{\text {nd }}$ one at 4-8 wks of age. 5- Live body weight, carcass, gizzard, and heart weights differed $(\mathrm{p}<0.01)$ in birds of the $3^{\text {rd }}$ generation compared to that of the $2^{\text {nd }}$ one at 16 and 20 wks of age. On the other hand, upgraded group had the highest $(p<0.01)$ values of most of carcass studied traits compared to the others, while the control one had significantly $(\mathrm{p}<0.01)$ the heaviest liver. It could be concluded that upgrading through crossing Silver Montazah sires to Baheij strain dams surpassed the selection in Baheij strain at 8 weeks of age throughout three generations in improving growth traits, chick viability, and carcass traits of progeny of both F2 and F3.
\end{abstract}

Key words: Upgrading- Selection-Growth and carcass traits- Body conformation- Chicks viability 


\section{INTRODUCTION}

Upgrading has most often been in "third world" countries to improve indigenous stocks, in so far as the local climatic and disease conditions allow. Highly production American and European poultry (or other livestock) often sicken and die in the tropics, but crosses of three-quarter bred with local breeds give a practical compromise between productivity and survivability (Scrivener, 2002). Baheij breed was developed through crossing with Silver Montazah strain (Mahmoud et al., 1974 and 1979). Therefore, crossing selected Silver Montazah males with Baheij females had to be applied in order to overcome the problem of reaching genetic equilibrium in Baheij breed, thus allowed effective selection for certain productive traits. Growth is a compound trait influenced by genetic and management, especially nutrition and health. Genetic improvement in growth of poultry has traditionally proceeded via selection for body weight at a fixed age. The estimates of genetic parameters provide support to analyze the genetic associations between traits in a data set. These estimates could be used to decide the selection method and choose what birds could be selected to attain the breeding goal (Ledur et al., 1993). Also, Knowledge on genetic parameters is essential for any genetic improvement program for growth or egg production. There is a lot of literature on genetic parameters for growth traits (Gondwe and Wollny, 2005; and Norris and Ngambi, 2006). Crossing was found to be effective for improving body weight as reported by Shebl et al. (1995), Nawar et al. (2004), and Amin (2007), who found positive heterosis in body weight at 6 and 12 wks of age in both sexes for two native Egyptian chicken, also, Nestor et al. (2006) and Amin (2009) reported positive effect of backcrossing on growth traits in turkey. Moreover, Mostafa and Nofal (2000), Amin (2009) found significant difference between the two sexes in body weight at different ages. On the other hand, correlations among the live body weight and body measurements and slaughter traits are important in poultry breeding. Isguzar (2003) reported that differences of body parts weights were statistically significant $(p<0.05)$ between genotypes and between sexes of Bronze and White turkeys. Moreover, the average percentages of wings, neck and head of females Bronze turkeys were higher than White turkeys at 14 and 18 weeks of age. In contrast, Ramakrishna et al. (2012) found that readyto-cook yield in tom turkeys did not differ significantly between three studied turkey varieties. Crossbreeding was found to improve chick viability (Nawar and Abdou, 1999; and Nawar et al., 2004). Information on the relationships among pre- and postslaughter traits of broiler chickens is valuable to poultry farmers and researchers as it allows early selection, as well as giving a chance to make an early evaluation of the breeding program (Yakubu et al., 2009). Amin (2009) using Gimmezah (G) strain as a sire strain in $G \times$ RIR cross resulted in superiority of eviscerated carcass weight and giblets weight percentages at 24 wks of age. Concerning the selection effect, Gaya et al. (2006) cited that based on the heritability estimates obtained, the analyzed traits (BW at $38 \mathrm{~d}$ and at $42 \mathrm{~d}$ and the carcass traits (eviscerated BW, breast and leg weights, and the body composition traits (heart, gizzard, liver weights) seemed to be able to respond to selection, at variable intensities. Sandercock et al. (2009) reported that the correlations for live weight, carcass yield, breast, drum, and wing portions were high; whereas those for the thigh portion and yield were low. Also, genetic variation for relative weight of heart was moderately high and greater at 10 than at 6 wk of age and broiler carcasses had a relatively smaller proportion of heart weight. The aim of the present study is to find out the effect of either upgrading or selection (for 8 weeks of age) programs for improving 
body weight at different ages, growth efficiency, and growth rate and chicks viability percentages at different intervals and both of body measurements and conformations at 8 and 12 wks of age, also, carcass traits at 16 and 20 wks of age were studied.

\section{MATERIALS AND METHODS}

This study was conducted at El-Sabahia Poultry Research Station, Alexandria, Animal Production Research Institute, Agriculture Research Center, Egypt.

\section{Breeding and management:}

Upgrading Line: In the first generation, females of the Baheij (Bj) breed were crossed to selected Silver Montazah (SM) males as a parent line according to the individual mature BW (average), thus, the females produced $(1 / 2 \mathrm{SM}+1 / 2 \mathrm{Bj})$ were backcrossed to the same parent line throughout two additional generations [produced $(3 / 4 \mathrm{SM}+1 / 4 \mathrm{Bj})$ and $(7 / 8 \mathrm{SM}+$ $1 / 8 \mathrm{Bj})$, in the two generations, respectively].

Selection Line: Selection scheme was accomplished to improve body weight at 8 weeks of age of Baheij breed throughout the three studied generations. On the basis of 8-week body weight birds were divided into heavy birds (mean \pm 0.5 standard deviation).

Control line: Chicks of Baheij breed were randomly chosen to establish a pedigreed control population.

Continuous lighting was provided from hatching to $8 \mathrm{wk}$ of age, at that time, the photoperiod was reduced to $12 \mathrm{~h} / \mathrm{d}$. and remained at this level during the rearing period. After the rearing period, at 20 weeks of age, females were housed in breeding pens (10 pullets +1 male, each). The birds were fed a starter diet (19\% crude protein and $2800 \mathrm{Kcal} / \mathrm{kg}$ ) up to 8 weeks of age, grower diet $(15 \%$ crud protein and $2700 \mathrm{~K} \mathrm{cal} / \mathrm{kg}$ ) up to 20 weeks (17\% crude protein and $2850 \mathrm{Kcal} / \mathrm{kg}$ ). Thereafter, feed and water were supplied ad libitum. The average number of progeny reared in the three generations was 68,135 ,
133 chick for the $\mathrm{BjBj}$ line, and 210,315 , 329 chick for the $\mathrm{SM} \times \mathrm{Bj}$ genotype, respectively.

\section{The studied traits:}

- Body weights (BW) at hatch, 4, 8, 12, 16, and 20 wks of age presented in Tables.

- Growth rate using the following equation (Lerner and Asmundson, 1932).

$\mathrm{GR}=\left[\mathrm{W}_{2}-\mathrm{W}_{1} / 1 / 2\left(\mathrm{~W}_{2}+\mathrm{W}_{1}\right)\right] \times 100$

Where: GR: rate of growth, W1: the initial weight, W2: the second weight.

- Chick viability through the growth period (0-8 weeks) of age.

- Body measurements:

Shank length, keel length and breast width which was measured with a modified verger angle meter in millimeters. It was measured at a point very near to the front end of the breast bone and $3 / 4$ inch down from the keel at 8 and 12 weeks of age at the $3^{\text {rd }}$ generation. Random sample of 6 cocks at 16 and 20 weeks of age from each genotype for the two generations were used to study the carcass traits (Absolute values and percentages of carcass, legs, gizzard liver and heart.

\section{Statistical analysis:}

Data of growth traits were analyzed using fixed models SAS institute (1988):

$\mathrm{Y}_{\mathrm{ijkl}}=\mathrm{U}+\mathrm{Gn}_{\mathrm{i}}+\mathrm{Gt}_{\mathrm{j}}+\mathrm{S}_{\mathrm{k}}+(\mathrm{GnGt})_{\mathrm{ij}}$ $\left.+(\mathrm{GnS})_{\mathrm{ik}}+(\mathrm{GtS})_{\mathrm{jk}}+\mathrm{GnGtS}\right)_{\mathrm{ijk}}+\mathrm{e}_{\mathrm{ijk}}$.

Where: $\mathrm{Y}_{\mathrm{ijkl}}=$ an observations, $\mathrm{U}=$ overall mean, $\mathrm{Gn}_{\mathrm{i}}=$ the fixed effect of $\mathrm{i}^{\text {th }}$ generation, $\mathrm{Gt}_{\mathrm{j}}=$ the fixed effect of $\mathrm{j}^{\text {th }}$ genotype, $S_{k}=$ the fixed effect of $k^{\text {th }}$ sex, and $(\mathrm{GnGt})_{\mathrm{ij}},(\mathrm{GnS})_{\mathrm{ik}},(\mathrm{GtS})_{\mathrm{jk}}$ and $(\mathrm{GnGtS})_{\mathrm{ijk}}=$ effects of the interactions between the three factors studied, and $\mathrm{e}$ ijkl $=$ random error.

The other traits which studied were analyzed using fixed models SAS institute (1988):

$\mathrm{Y}_{\mathrm{ijk}}=\mathrm{U}+\mathrm{Gn}_{\mathrm{i}}+\mathrm{Gt}_{\mathrm{j}}+(\mathrm{GnGt})_{\mathrm{ij}}+\mathrm{e}_{\mathrm{ijk}}$

Where: $\mathrm{Y}_{\mathrm{ijk}}=$ an observations, $\mathrm{U}=$ overall mean, $\mathrm{Gn}_{\mathrm{i}}=$ the fixed effect of $\mathrm{i}^{\text {th }}$ generation, $\mathrm{Gt}_{\mathrm{j}}=$ the fixed effect of $\mathrm{j}^{\text {th }}$ genotype, $(\mathrm{GnGt})_{\mathrm{ij}}=$ effect of the interaction between the two main factors, and e $\mathrm{ijk}=$ random error. Significant differences 
among means were tested by Duncan Test (1955).

\section{RESULTS AND DISCUSSION}

1- Body weight (BW): Least square means for body weight (BW) as affected by generation, genetic group for males and females are presented in Table 1. Concerning the effect of generation, the results showed that, chicks of the $3^{\text {rd }}$ generation were the heaviest $(\mathrm{p}<0.001)$ compared to those of the $1^{\text {st }}$ and $2^{\text {nd }}$ one (801, 1068 and $1298 \mathrm{gm}$ ) at 12, 16 and 20 wks of age, respectively. The same trend was found at the early periods of growth $(0,4$, and 8 wks of age as shown in Table $1)$. On the other hand, while the average of body weight of both of the $1^{\text {st }}$ and $2^{\text {nd }}$ generations were nearly similar at $12 \mathrm{wks}$ of age, the superiority of the $1^{\text {st }}$ one over the $2^{\text {nd }}$ generation $(p<0.001)$ was found at 16 wks of age, while the opposite was found at 20 wks of age. Also, results showed that the least square means of chicks BW of upgraded group surpassed $(p<0.001)$ those of selected and control groups at all ages studied. At the meantime, the selected group surpassed $(p<0.001)$ those of control one at all ages studied.

The positive effect of crossing agreed with the finding of Yalcin et al. (2000), Mohamed (2003), Aly et al. (2005), and Amin (2007) on chicken. In addition, Aly et al. (2006) reported that heterotic effects of crossing between Bandarah (B) x G cocks and their reciprocal crosses were positive in body weight at 16 weeks of age. Moreover, Nestor et al. (2006) reported that for maximum gains per generation, backcrossing probably should be used for maximum of two or three generations. Amin (2009) found that using $G$ strain as a dam strain in $G \times$ Kosmos (KK) and RIR $x$ KK crosses improved body weight rate at 4, 8, 12 and 16 wks of age.

As for the effect of selection on BW, selection for high and low threshold weight for onset of lay, carried out in a broiler line, the total response to selection over two generations was $382 \mathrm{~g}$. The difference in 6wk body weight of the two lines was $+19 \mathrm{~g}$ in favor ( $p>0.05)$ of the high line (Eitan and Soller, 1995). Williams et al. (2002) found that long-term divergent selection for high $(\mathrm{HH})$ and low (LL) BW at $56 \mathrm{~d}$ of age in White Plymouth Rock chickens resulted in superiority of line $\mathrm{HH}$ chickens of the $42^{\text {nd }}$ generation of selection, they were heavier at $28,56,168$, and $266 \mathrm{~d}$ of age, as well as at sexual maturity than those from line LL of the same generation. Nestor et al. (2008) reported that a line $(\mathrm{F})$ of turkeys was selected over 40 generations for increased 16-wk BW. Selection was effective in increasing 16-wk BW and genetic increases in 16-wk BW in the selected line were positively associated with $\mathrm{BW}$ at other ages (8 and 20 wks of age and at $50 \%$ production), shank length at $16 \mathrm{wk}$. of age. Moreover, Nigussie et al. (2011) found that the BW16 showed higher heritability, this trait seemed to have common genes and utilizing it as selection trait would be expected to improve growth performance of local studied chicken.

Males were heavier $(p<0.001)$ than female at all ages studied except those of 1 day of hatch which had nearly similar weight averages. Same results were reported by Mostafa and Nofal (2000) and Amin (2008). Statistical analysis revealed no significant interaction between the three main factors except in BW at 8, 16 and 20 wks of age. Significant $(p<0.001)$ effect of interaction between generation and genotype was found. The birds in the $3^{\text {rd }}$ generation $(7 / 8 \mathrm{SM}+1 / 8 \mathrm{Bj})$ were the heaviest (1171 and $1419 \mathrm{~g})$ at 16 and 20 weeks of age, respectively.

2-Growth rate percentage (GR\%): The results presented in Table 2 showed that least square means of GR\% of birds differed $(\mathrm{p}<0.001)$ through the three consecutive generations, where birds of the $3^{\text {rd }}$ Generation had the highest value of GR\% through the intervals 0-12, 12-16, 12$20,0-16$ and $0-20$ wks of age $(182.4,28.0$, 
46.8, 186.5 and $188.8 \%$, respectively), except that of the late age (16-20 wks), the $2^{\text {nd }}$ generation had the highest value (24.7\%).

Both of the upgraded and the selected groups had significantly the highest values compared with the control one at 0-12, 1220 and at $0-16$ wks of age, while both of the upgraded and control group surpassed $(p<0.001)$ the selected one at $12-16$ wks at age. No significant differences were found within the three genotypes at 16-20 and 020 wks of age. Barbato (1991) found on meat-type chickens that growth rate exhibited significant heterosis due to both autosomes and the sex chromosomes. Highly significant differences were found between the two sexes at $0-12,12-16,12-$ 20, 0-16 and 0-20 wks of age where males had higher growth rate percentage than female. These results were in agreement with those reported by Mostafa and Nofal (2000) and Amin (2008 and 2009) who found significant differences between both sexes in body weight. All interactions between the main factors were not significant except that for GR\% at 0-16 wks of age. The birds of upgraded groups at both the $1^{\text {st }}$ and $3^{\text {rd }}$ generations were equal and had significant GR\% (185.5\%).

3- Body measurements (BM): Results presented in Table 3 suggested BM of birds differed by age where, body weight (BW), shank length (SL), keel length (KL) and breast width (BW) had significantly $(p<0.01)$ higher average at 12 wks of age compared to those at $8 \mathrm{wks}$ of age. Also, BW and SL differed significantly $(\mathrm{p}<0.05)$ within the three genotypes where, the selected group had the highest BW while, the upgraded group had the highest average of SL. Moreover, male had significantly higher values of BW $(\mathrm{p}<0.01)$ than females $(750.1$ vs. $576.3 \mathrm{~g})$. These results were in agreement with those reported by ElTurkey (1981) who reported that $3^{\text {rd }}$ generations were nearly similar and higher than that of the $2^{\text {nd }}$ one at 4-8 wks of age. On the other hand, no significant differences were not significant among body measurements of purebreds and crossbreds at certain ages (8 and 12 wks), while Abou-El-Ella (1982) reported that crossbreeding was found to be effective on body measurements, particularly on keel length and breast width. In addition, Amin (2009) found that all crosses and backcrosses in turkey had significantly the lowest means of growth efficiency through the period (4-20 wks of age) concerning the three generations studied and the difference between overall means of growth efficiency for both sexes was significant at all periods studied except for 12-16 and 12-20 wks of age. On the other hand, our results disagreed with those reported by Sharaf et al. (2006) concerning both shank and keel lengths at 4 weeks in quail, while the results of the same authors concerning both traits showed that average of reciprocal crossbreds surpassed both of pure and crossbred averages at 5 wks of age. General increase in some body measurements in each genotype as age increased and this finding in agreement with Adedeji et al. (2006). Mulder et al. (2009) results indicate good opportunities to simultaneously increase the mean and improve uniformity of body weight of broilers by selection. Wolc et al. (2009) was indicating the possibility of improving uniformity of BW (34-d-old male and female broiler chickens) and conformation by means of selection and genetic correlations for BW, and for conformation score, between sexes were high. Rekaya (2013) reported that genetic correlations between BW and conformation traits were moderate to high.

4-Viability percentage (V\%): Results in Table 4 showed that viability of chicks differed significantly $(\mathrm{p}<0.01)$ through the period 0-4 and 4-8 wks of age for the three generations studied where the viability of the $2^{\text {nd }}$ generation was the highest at 0-4 wks of age, while the values of the $1^{\text {st }}$ and differences were found within the three genotypes, also the same results were found between the two sexes at the different 
period studied. The results of this study disagreed with those reported for some investigators who confirmed the superiority of crossbred over purebreds in viability (Fairfull, 1990; Mandour et al., 1992; Aly et al., 2005; and Amin, 2007).

5-Carcass traits: Results in Table 5 showed that live body weight, carcass, gizzard, and heart weights differed significantly $(\mathrm{p}<0.01)$ in birds of the $3^{\text {rd }}$ generation compared to that of the $2^{\text {nd }}$ one at 16 and 20 wks of age (1331.6 g, 1044.6 g, $40.14 \mathrm{~g}$, and $11.38 \mathrm{~g}$ vs. $1126.8 \mathrm{~g}, 836.1$ g, 35.03g, and $8.68 \mathrm{~g}$, respectively). Concerning leg and liver weights, no significant differences were found between the two generations studied. On the other hand, upgraded group had the highest $(p<0.01)$ live weight, carcass, gizzard and heart weights compared to the others while the control group had significantly the heaviest liver. No significant effects of genotype on percentages of the studied organs. These results were in agreement with those reported by several investigators. Mandour et al. (1996) reported that the overall mean of edible giblets percentages were greater $(p<0.05)$ for Silver Montazah line crosses than corresponding pure strain. Aly et al. (2006) reported that liver relative weight of $B \times \mathrm{G}$ cross cocks was the heaviest, also, negative heterotic effects were found concerning relative weights of most organs studied. It was found that the lowest dressing percentage was obtained when Silver Montazah sire mated to different dams (ElTurkey, 1981). Moreover, Balat et al.
(2005) reported that carcass traits did not express any significant differences between purebreds $\mathrm{Bj}$, Matrouh (Mat) and White Leghorn (WL) and crossbred (Bj x Mat. or Bj $\mathrm{x}$ WL), where Baheij breed was used as a sire. Moreover, Amin (2009) found heterotic effects of the reciprocal crosses among G, RIR, and KK strains which were positive for drawing weight and for eviscerated carcass weight at 24 wks of age. On the other hand, Konarzewski et al. (2000) reported that the differences between strains in growth rate during the first week after hatching were not reflected in similar differences in the relative masses of the heart, liver. Cloete et al. (2006) on ostrich, reported that heritability estimate was small (0.12) for chest circumference. Sandercock et al. (2009) found that the relative heart weight decreases with selection for body weight as has been reported by several authors (e.g., Jackson and Diamond, 1996; Rance et al., 2002; and Gaya et al., 2007). Also, Gaya et al. (2006) reported that the genetic correlation estimates between body composition traits were variable. A large genetic association between a great number of performance and carcass traits seemed to exist. It could be concluded that upgrading line through crossing Silver Montazah sires to Baheij strain dams surpassed the selected line in Baheij breed for body weight at 8 weeks of age throughout three generations, in improving most of the growth traits, certain body conformation, chicks viability, and carcass traits of progeny of both the second and the third generations which studied. 
Table (1): Effect of genotype and generation on least squares means of body weight of male and female birds at different ages studied

\begin{tabular}{|c|c|c|c|c|c|c|c|c|c|c|}
\hline \multirow{3}{*}{\multicolumn{2}{|c|}{\begin{tabular}{|l|l|} 
& \\
Generation & Genotype
\end{tabular}}} & \multicolumn{9}{|c|}{ Body weight, $g$} \\
\hline & & \multicolumn{3}{|c|}{ 1-day } & \multicolumn{3}{|c|}{ 4-wk } & \multicolumn{3}{|c|}{ 8-wk } \\
\hline & & $\mathbf{M}$ & $\mathbf{F}$ & Average & $\mathbf{M}$ & $\mathbf{F}$ & Average & $\mathbf{M}$ & $\mathbf{F}$ & Average \\
\hline \multirow{3}{*}{1} & $1 / 2 \mathrm{SM} x 1 / 2 \mathrm{Bj}$ & $34.7 \pm 0.3$ & $34.1 \pm 0.3$ & $34.4 \pm 0.2$ & $274.3 \pm 5.7$ & $255.9 \pm 6.8$ & $265.1 \pm 4.5$ & $460.7 \pm 8.2$ & $414.6 \pm 9.7$ & $437.7 \pm 6.3$ \\
\hline & $\begin{array}{l}\text { Bj.Selected } \\
\text { L1 }\end{array}$ & $34.3 \pm 0.3$ & $34.9 \pm 0.3$ & $34.1 \pm 0.2$ & $252.7 \pm 6.1$ & $236.7 \pm 6.9$ & $244.7 \pm 4.6$ & $435.6 \pm 8.7$ & $372.5 \pm 9.9$ & $404.0 \pm 6.6$ \\
\hline & $\mathrm{Bj} \times \mathrm{Bj}$ & $34.2 \pm 0.4$ & $34.7 \pm 0.4$ & $33.9 \pm 0.3$ & $238.9 \pm 9.8$ & $215.4 \pm 6.8$ & $277.1 \pm 6.8$ & $411.1 \pm 14.0$ & $356.6 \pm 13.8$ & $383.9 \pm 9.8$ \\
\hline \multicolumn{2}{|c|}{ Overall mean } & $34.4 \pm 0.2$ & $33.9 \pm 0.2$ & $34.1 \pm 0.1^{\mathrm{B}}$ & $255.3 \pm 4.3$ & $236.0 \pm 4.5$ & $245.6 \pm 3.1^{\mathrm{C}}$ & $435.8 \pm 6.1$ & $381.3 \pm 6.6$ & $408.5 \pm 4.5^{\mathrm{C}}$ \\
\hline \multirow{3}{*}{2} & 3/4SMx1/4Bj & $34.5 \pm 0.2$ & $34.1 \pm 0.2$ & $34.3 \pm 0.1$ & $289.3 \pm 4.7$ & $246.2 \pm 4.9$ & $267.8 \pm 3.4$ & $519.2 \pm 7.0$ & $429.3 \pm 7.6$ & $474.2 \pm 5.1$ \\
\hline & $\begin{array}{l}\text { Bj.Selected } \\
\text { L2 }\end{array}$ & $34.1 \pm 0.2$ & $33.6 \pm 0.3$ & $33.9 \pm 0.2$ & $269.9 \pm 5.0$ & $239.2 \pm 5.2$ & $254.5 \pm 4.6$ & $499.1 \pm 7.3$ & $407.1 \pm 9.9$ & $453.1 \pm 5.3$ \\
\hline & $\mathrm{Bj} \times \mathrm{Bj}$ & $34.1 \pm 0.4$ & $33.4 \pm 0.3$ & $33.7 \pm 0.3$ & $256.6 \pm 8.1$ & $234.4 \pm 7.0$ & $245.5 \pm 5.4$ & $441.6 \pm 14.0$ & $392.2+10.2$ & $416.9 \pm 7.8$ \\
\hline \multicolumn{2}{|c|}{ Overall mean } & $34.2 \pm 0.2$ & $33.7 \pm 0.2$ & $34.0 \pm 0.1^{\mathrm{B}}$ & $271.9 \pm 0.3 .5$ & $240.0 \pm 3.3$ & $255.9 \pm 2.5^{\mathrm{B}}$ & $486 . \overline{6} \pm 5.1$ & $409 . \overline{5 \pm 4} .9$ & $448.1 \pm 3.6^{\mathrm{B}}$ \\
\hline \multirow{3}{*}{3} & 7/8SMx 1/8Bj & $36.0 \pm 0.2$ & $36.6 \pm 0.2$ & $36.3 \pm 0.1$ & $307.0 \pm 3.9$ & $247.9 \pm 4.7$ & $277.5 \pm 3.0$ & $628.4 \pm 5.6$ & $506.4 \pm 6.8$ & $567 . \overline{4} \pm 4.4$ \\
\hline & $\begin{array}{l}\text { Bj.Selected } \\
\text { L3 }\end{array}$ & $36.0 \pm 0.2$ & $36.4 \pm 0.2$ & $36.2 \pm 0.2$ & $266.9 \pm 5.1$ & $271.4 \pm 5.4$ & $269.2 \pm 3.7$ & $638.9 \pm 7.3$ & $525.0 \pm 7.8$ & $581.9 \pm 5.3$ \\
\hline & $\mathrm{Bj} \mathrm{xBj}$ & $34.9 \pm 0.8$ & $34.2 \pm 0.7$ & $34.6 \pm 0.5$ & $254.8 \pm 16.0$ & $223.9 \pm 14.0$ & $239.3 \pm 11.1$ & $462.2 \pm 2.3$ & $402.7 \pm 20.9$ & $432.4 \pm 15.9$ \\
\hline \multicolumn{2}{|c|}{ Overall mean } & $35.7 \pm 0.3$ & $35.7 \pm 0.3$ & $35.7 \pm 0.1^{\mathrm{A}}$ & $276.2 \pm 6.0$ & $247.7 \pm 5.0$ & $262.0 \pm 4.6^{\mathrm{A}}$ & $576.5 \pm 4.5$ & $478.0 \pm 7.8$ & $527.3 \pm 5.8^{\mathrm{A}}$ \\
\hline \multirow{3}{*}{\multicolumn{2}{|c|}{$\begin{array}{l}\text { Crossbreds overall mean } \\
\text { Bj.Selected overall mean } \\
\text { Baheij overall mean } \\
\end{array}$}} & $35.0 \pm 0.1$ & $34.9 \pm 0.2$ & $35.0 \pm 0.1^{\mathrm{a}}$ & $290.2 \pm 2.8$ & $250.0 \pm 3.0$ & $270.1 \pm 2.1^{\mathrm{a}}$ & $536.1 \pm 4.0$ & $450.1 \pm 4.6$ & $493.1 \pm 3.1^{\mathrm{a}}$ \\
\hline & & $34.8 \pm 0.2$ & $34.6 \pm 0.2$ & $34.7 \pm 0.1^{\mathrm{b}}$ & $263.2 \pm 3.1$ & $249.1 \pm 3.4$ & $256.1 \pm 2.3^{b}$ & $524.5 \pm 4.5$ & $534.9 \pm 4.9$ & $479.7 \pm 3.3^{\mathrm{b}}$ \\
\hline & & $34.4 \pm 0.3$ & $33.8 \pm 0.3$ & $34.1 \pm 0.1^{\mathrm{c}}$ & $250.1 \pm 7.0$ & $224.6 \pm 6.3$ & $237.3 \pm 4.7^{\mathrm{c}}$ & $438.3 \pm 10.0$ & $383.8 \pm 9.1$ & $411.1 \pm 6.7^{\mathrm{c}}$ \\
\hline \multicolumn{2}{|c|}{ Genotypes overall mean } & $34.8+0.1$ & $34.4+0.1$ & 34.87 & $267.8+2.7$ & $241.2+2.6$ & $26 \overline{3.5}$ & $499 . \overline{6}+3.9$ & $422.9+3.7$ & $49 \overline{2.4}$ \\
\hline
\end{tabular}

- Means having different letters in every column and within every factor of treatments are significantly different $(\mathrm{p}<0.05)$.

- All main factors studied had highly significant (0.001) effect on the body weight except the sex which had no significant effect on body weight at hatch, while all interactions between them were not significant. 
Cont. Table (1).

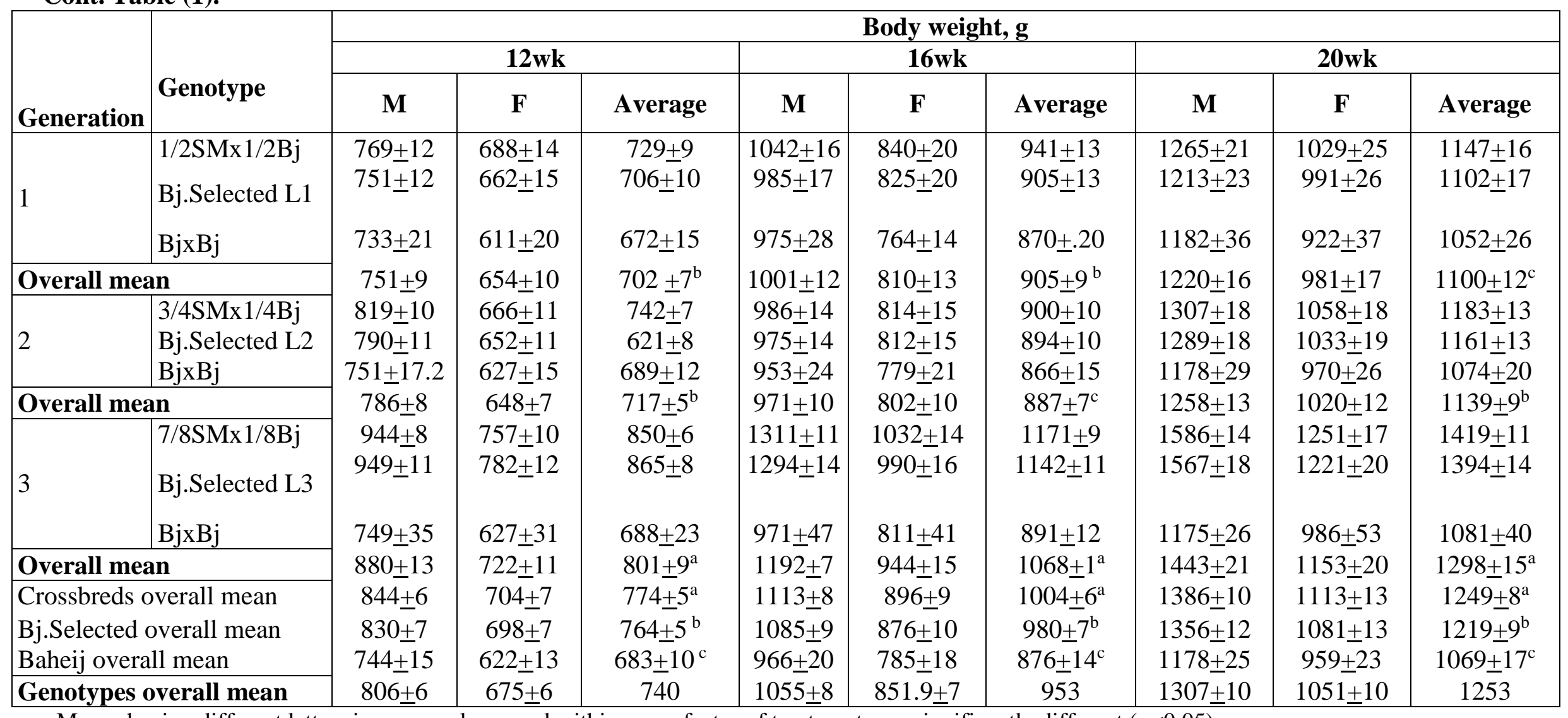

- Means having different letters in every column and within every factor of treatments are significantly different $(p<0.05)$.

- All main factors studied had highly significant (0.001) effect on the body weight while all interactions between them were not significant except that for body weight at 16 and 20 wks of age, were influenced $(p<0.001)$ by the interaction of generation $x$ genotype, 
Table (2): Effect of genotype and generation on least squares means of growth rate percentage of male and female birds at different ages studied

\begin{tabular}{|c|c|c|c|c|c|c|c|c|c|c|}
\hline \multirow{3}{*}{ Generation } & \multirow{3}{*}{ Genotype } & \multicolumn{9}{|c|}{ Growth rate percentage } \\
\hline & & \multicolumn{3}{|c|}{ 0-12 wks } & \multicolumn{3}{|c|}{ 12-16 wks } & \multicolumn{3}{|c|}{ 16-20 wks } \\
\hline & & $\mathbf{M}$ & $\mathbf{F}$ & Average & $\mathbf{M}$ & $\mathbf{F}$ & Average & $\mathbf{M}$ & $\mathbf{F}$ & Average \\
\hline \multirow[t]{3}{*}{1} & $1 / 2 \mathrm{SMx} 1 / 2 \mathrm{Bj}$ & $180.9 \pm 0.6$ & $180.4 \pm 0.5$ & $180.7 \pm 0.3$ & $30.1 \pm 1.0$ & $20.6 \pm 1.2$ & $25.33 \pm 0.8$ & $20.3 \pm 0.9$ & $21.3 \pm 1.2$ & $20.8 \pm 0.7$ \\
\hline & Bj.Selected L1 & $182.1 \pm 0.5$ & $179.7 \pm 0.5$ & $180.9 \pm 0.3$ & $26.5 \pm 1.1$ & $23.9 \pm 1.2$ & $25.2 \pm 0.8$ & $21.9 \pm 1.2$ & $20.9 \pm 1.1$ & $21.4 \pm 0.8$ \\
\hline & $\mathrm{Bj} \mathrm{Bj}$ & $181.7+0.9$ & $178.3+0.4$ & $180.0+0.6$ & $28.5+1.7$ & $24.1+1.7$ & $26.3+1.2$ & $20.1+0.8$ & $22.6+1.8$ & $21.4+1.1$ \\
\hline \multicolumn{2}{|c|}{ Overall mean } & $181.6+0.3$ & $179.5 \pm 0.4$ & $180.5 \pm 0.3^{\mathrm{C}}$ & $28.4 \pm 0.7$ & $22.8 \pm 0.8$ & $25.6 \pm 0.6^{\mathrm{b}}$ & $20.7 \pm 0.8$ & $21.6 \pm 0.3$ & $21.2 \pm 0.5^{\mathrm{b}}$ \\
\hline \multirow[t]{3}{*}{2} & 3/4SMx1/4Bj & $183.4 \pm 0.4$ & $179.9 \pm 0.4$ & $181 . \overline{6}+0.4$ & $19.1 \pm 0.9$ & $21.2 \pm 0.5$ & $20.1 \pm 0.6$ & $28.1 \pm 0.8$ & $25.5 \pm 0.8$ & $26.8+0.5$ \\
\hline & Bj.Selected L2 & $182.9 \pm 0.4$ & $179.8 \pm 0.4$ & $181.4 \pm 0.3$ & $21.2 \pm 0.8$ & $21.9 \pm 0.9$ & $21.6 \pm 0.6$ & $28.1 \pm 0.9$ & $22.8 \pm 0.9$ & $25.4+0.6$ \\
\hline & $\mathrm{Bj} \times \mathrm{Bj}$ & $182.4 \pm 0.6$ & $179.2 \pm 0.6$ & $180.8 \pm 0.5$ & $23.4 \pm 1.4$ & $22.3 \pm 1.3$ & $22.9 \pm 0.9$ & $21.7 \pm 1.4$ & $22.2 \pm 1.3$ & $22.0+0.8$ \\
\hline \multicolumn{2}{|c|}{ Overall mean } & $182.9+0.3$ & $179.6 \pm 0.2$ & $181.3 \pm 0.2^{\mathrm{b}}$ & $21.2 \pm 0.6$ & $21.7 \pm 0.6$ & $21.5 \pm 0.4^{\mathrm{c}}$ & $25.9 \pm 0.6$ & $23.5 \pm 0.6$ & $24.7 \pm 0.4^{\mathrm{a}}$ \\
\hline \multirow[t]{3}{*}{3} & $7 / 8 \mathrm{SMx} 1 / 8 \mathrm{Bj}$ & $185.1 \pm 0.3$ & $181.2 \pm 0.3$ & $183.1 \pm 0.3$ & $32.0 \pm 0.6$ & $30.1 \pm 0.8$ & $31.0 \pm 0.6$ & $18.5 \pm 0.7$ & $19.0 \pm 0.8$ & $18.8+0.5$ \\
\hline & Bj.Selected L3 & $185.2 \pm 0.4$ & $182 \pm 0.4$ & $183.5 \pm 0.3$ & $30.4 \pm 0.9$ & $23.4 \pm 2.9$ & $26.9 \pm 0.6$ & $19.0 \pm 0.9$ & $20.7 \pm 1.0$ & $19.8+0.6$ \\
\hline & $\mathrm{Bj} \times \mathrm{Bj}$ & $181.7 \pm 1.3$ & $179 . \overline{1} \pm 1.7$ & $180.4 \pm 1.1$ & $26.6 \pm 2.9$ & $25.7 \pm 2.6$ & $26.2 \pm 2.0$ & $19.2 \pm 2.5$ & $19.6 \pm 2.3$ & $19.4+1.7$ \\
\hline & Overall mean & $183.9 \pm 0.4$ & $180.8 \pm 0.4$ & $182.4 \pm 0.3^{\mathrm{a}}$ & $29.7 \pm 1.0$ & $26.4 \pm 0.9$ & $28.0 \pm 0.7^{\mathrm{a}}$ & $18.9 \pm 1.0$ & $19.8 \pm 1.9$ & $19.3 \pm 0.7^{\mathrm{c}}$ \\
\hline & $183.1 \pm 0.3$ & $180.5 \pm 0.2$ & $181.8 \pm 0.2$ & $27.1 \pm 0.5$ & $23.9 \pm 0.5$ & $25.5 \pm 0.3$ & $22.3 \pm 0.5$ & $21.9 \pm 0.5$ & $22.1 \pm 0.3$ \\
\hline \multicolumn{2}{|c|}{$\begin{array}{l}\text { Crossbreds overall mean } \\
\text { Bj.Selected line overall } \\
\text { mean }\end{array}$} & $183.4 \pm 0.2$ & $180.5 \pm 0.2$ & $181.9 \pm 0.2$ & $26.1 \pm 0.5$ & $23.1 \pm 0.6$ & $24.6 \pm 0.4$ & $22.9 \pm 0.5$ & $21.4 \pm 0.6$ & $22.2 \pm 0.4$ \\
\hline \multirow{2}{*}{\multicolumn{2}{|c|}{ Baheij overall mean }} & $181.9 \pm 0.5$ & $178.8 \pm 0.5$ & $180.4 \pm 0.5$ & $26.2 \pm 1.2$ & $24.0 \pm 1.1$ & $25.1 \pm 0.8$ & $20.4 \pm 1.1$ & $21.5 \pm 1.0$ & $20.9 \pm 0.7$ \\
\hline & & $182.8 \pm 0.23$ & $179.9 \pm 0.20$ & $182 . \overline{0} 1$ & $26.4 \pm 0.4$ & $23.7 \pm 0.4$ & $25 . \overline{4} 6$ & $21.9 \pm 0.5$ & $21.6 \pm 0.5$ & $22 . \overline{0} 0$ \\
\hline \multicolumn{11}{|c|}{ Significance of: } \\
\hline \multirow{2}{*}{\multicolumn{2}{|c|}{$\begin{array}{l}\text { Generation }(\mathrm{Gn}) \\
\text { Genotype (Gt) }\end{array}$}} & \multicolumn{3}{|l|}{$* * *$} & \multicolumn{3}{|l|}{$* * *$} & \multicolumn{3}{|l|}{$* * *$} \\
\hline & & \multirow{2}{*}{\multicolumn{3}{|c|}{ ** }} & \multicolumn{3}{|l|}{$* *$} & \multicolumn{3}{|l|}{ NS } \\
\hline \multicolumn{2}{|c|}{$\operatorname{Sex}(\mathrm{S})$} & & & & \multicolumn{3}{|l|}{$* * *$} & \multicolumn{3}{|l|}{ NS } \\
\hline
\end{tabular}

- Means having different letters in every column and within every factor of treatments are significantly different $(\mathrm{p}<0.05)$,

- All interactions between the main factors were not significant except that for growth rate $\%$ at $0-16$ period, which was influenced (p<0.001) by the interaction of generation $\mathrm{x}$ genotype,

** Significant at $\mathrm{p}<0.01, * * *$ Significant at $\mathrm{p}<0.001, \mathrm{NS}$ : non significant. 
Cont. Table (2).

\begin{tabular}{|c|c|c|c|c|c|c|c|c|c|c|}
\hline \multirow{3}{*}{ Generation } & \multirow{3}{*}{ Genotype } & \multicolumn{9}{|c|}{ Growth rate percentage } \\
\hline & & \multicolumn{3}{|c|}{ 12-20 wks } & \multicolumn{3}{|c|}{ 0-16 wks } & \multicolumn{3}{|c|}{ 0-20 wks } \\
\hline & & $\mathbf{M}$ & $\mathbf{F}$ & Average & $\mathbf{M}$ & $\mathbf{F}$ & Average & $\mathbf{M}$ & $\mathbf{F}$ & Average \\
\hline \multirow{3}{*}{1} & $1 / 2 \mathrm{SMx} 1 / 2 \mathrm{Bj}$ & $50.8 \pm 1.4$ & $41.3 \pm 1.8$ & $46.0 \pm 1.1$ & $186.9 \pm 0.2$ & $184.0 \pm 1.3$ & $185.5 \pm 0.2$ & $187.5 \pm 0.6$ & $186.9 \pm 0.7$ & $187.2+0.4$ \\
\hline & Bj.Selected L1 & $48.1 \pm 1.6$ & $44.2 \pm 1.8$ & $46.2 \pm 1.2$ & $186.3 \pm 0.2$ & $183.7 \pm 0.3$ & $184.9 \pm 0.2$ & $188.8 \pm 0.5$ & $186.5 \pm 0.6$ & $187.8+0.4$ \\
\hline & $\mathrm{Bj} \times \mathrm{Bj}$ & $46.5 \pm 2.5$ & $45.8 \pm 2.6$ & $46.1 \pm 1.7$ & $186.2 \pm 0.4$ & $182.7 \pm 0.4$ & $184.4 \pm 0.3$ & $188.6 \pm 1.9$ & $185.8 \pm 0.9$ & $187.2+0.7$ \\
\hline \multicolumn{2}{|c|}{ Overall mean } & $48.5+1.2$ & $43.7 \pm 1.2$ & $46.1 \pm 0.8^{\mathrm{b}}$ & $186.4 \pm 0.1$ & $183.5 \pm 0.2$ & $184.9 \pm 0.1^{\mathrm{b}}$ & $188.3 \pm 0.4$ & $186.4 \pm 0.4$ & $187.3 \pm 0.2^{b}$ \\
\hline \multirow{3}{*}{2} & 3/4SMx $1 / 4 \mathrm{Bj}$ & $46.4 \pm 1.1$ & $45.6 \pm 1.2$ & $46.0 \pm 0.8$ & $186.2+0.1$ & $183.6 \pm 0.2$ & $184.9 \pm 0.1$ & $189.6 \pm 0.5$ & $187.2 \pm 0.5$ & $188.4+0.3$ \\
\hline & Bj.Selected L2 & $48.5 \pm 1.3$ & $43.8 \pm 1.3$ & $46.2 \pm 0.9$ & $86.2 \pm 0.2$ & $183 . \overline{ \pm} 0.3$ & $184.9 \pm 0.1$ & $189.4 \pm 0.4$ & $186.2 \pm 0.4$ & $187.8+0.3$ \\
\hline & BjxBj & $44.6 \pm 1.9$ & $43.8 \pm 1.3$ & $44.2 \pm 1.3$ & $185.9 \pm 0.3$ & $183.2 \pm 0.3$ & $184.6 \pm 0.2$ & $188.7 \pm 0.8$ & $186.5 \pm 0.7$ & $187.6+0.5$ \\
\hline \multicolumn{2}{|c|}{ Overall mean } & $46.5+0.9$ & $44.4 \pm 0.8$ & $45.4 \pm 0.6^{\mathrm{b}}$ & $186.1 \pm 0.1$ & $183.5 \pm 0.1$ & $184.8 \pm 0.1^{\mathrm{b}}$ & $189.2 \pm 0.3$ & $186.6 \pm 0.1$ & $187.9 \pm 0.2^{\mathrm{b}}$ \\
\hline \multirow{3}{*}{3} & 7/8SMx1/8Bj & $49.7 \pm 0.9$ & $48.3 \pm 1.1$ & $49.0 \pm 0.7$ & $186.9 \pm 0.2$ & $184.0 \pm 0.3$ & $187.5 \pm 0.1$ & $187.5 \pm 0.6$ & $186.9 \pm 0.7$ & $187.2+0.4$ \\
\hline & Bj. Selected L3 & $48.6 \pm 1.2$ & $43.6 \pm 1.4$ & $46.1 \pm 0.9$ & $188.9 \pm 0.2$ & $185 . \overline{ \pm} 0.6$ & $187.3 \pm 0.1$ & $195.8 \pm 0.4$ & $188.3 \pm 0.4$ & $189.6+0.2$ \\
\hline & BjxBj & $45.2 \pm 3.9$ & $45.7 \pm 3.5$ & $45.4 \pm 2.6$ & $186.2 \pm 0.4$ & $182.7 \pm 0.4$ & $184.7 \pm 0.3$ & $188.6 \pm 0.9$ & $185.8 \pm 0.9$ & $187.2+0.7$ \\
\hline \multicolumn{2}{|c|}{ Overall mean } & $47.8+1.4$ & $45.9 \pm 1.3$ & $46.8 \pm 0.7^{\mathrm{a}}$ & $187.9 \pm 0.2$ & $185.1 \pm 0.2$ & $186.5 \pm 0.1^{\mathrm{a}}$ & $189.9 \pm 0.5$ & $187.7 \pm 0.5$ & $188.9 \pm 0.3^{\mathrm{a}}$ \\
\hline \multirow{2}{*}{\multicolumn{2}{|c|}{$\begin{array}{l}\text { Crossbreds overall mean } \\
\text { Bj.Selected line overall } \\
\text { mean }\end{array}$}} & $48.9+0.7$ & $45.0 \pm 0.8$ & $47.0 \pm 0.5$ & $187.4 \pm 0.1$ & $184.5 \pm 0.1$ & $186.0 \pm 0.1^{\mathrm{a}}$ & $189.3 \pm 0.3$ & $187.5 \pm 0.3$ & $188.4 \underline{ \pm} 0.2$ \\
\hline & & $48.4+0.8$ & $43.8 \pm 0.8$ & $46.2 \pm 0.6$ & $187.1 \pm 0.1$ & $184 . \overline{4} 01$ & $185.7 \pm 0.08$ & $189.7 \pm 0.3$ & $186.9 \pm 0.3$ & $188.3 \pm 0.2$ \\
\hline \multicolumn{2}{|c|}{ Baheij overall mean } & $45.4+1.6$ & $45.1 \pm 1.5$ & $45.3 \pm 1.6$ & $186.0 \pm 0.3$ & $183.2 \pm 0.2$ & $184.6 \pm 0.2 b$ & $188.6 \pm 0.7$ & $186.2 \pm 0.6$ & $187.4 \pm 0.4$ \\
\hline \multicolumn{2}{|c|}{$\begin{array}{l}\text { Overall mean of } \\
\text { genotypes }\end{array}$} & $47.6+0.6$ & $44.7 \pm 0.6$ & $46 . \overline{7} 7$ & $186.8 \pm 0.1$ & $184.0 \pm 0.1$ & 185.99 & $189.2 \pm 0.2$ & $186.9 \pm 0.2$ & $188 . \overline{5} 5$ \\
\hline \multicolumn{11}{|c|}{ Significance of: } \\
\hline \multicolumn{2}{|c|}{ Generation (Gn) } & \multicolumn{3}{|c|}{$* * *$} & \multicolumn{3}{|c|}{$* * *$} & \multicolumn{3}{|c|}{$* * *$} \\
\hline \multicolumn{2}{|c|}{ Genotype (Gt) } & \multicolumn{3}{|c|}{$*$} & \multicolumn{3}{|c|}{$* * *$} & \multicolumn{3}{|c|}{ NS } \\
\hline \multicolumn{2}{|c|}{$\operatorname{Sex}(\mathrm{S})$} & \multicolumn{3}{|c|}{$* * *$} & \multicolumn{3}{|c|}{$* *$} & \multicolumn{3}{|c|}{$* * *$} \\
\hline
\end{tabular}

- Means having different letters in every column and within every factor of treatments are significantly different $(\mathrm{p}<0.05)$,

- All interactions between the main factors were not significant except that for growth rate $\%$ at $0-16$ period, which was influenced (p<0.001) by the interaction of generation $\mathrm{x}$ genotype,

* Significant at $\mathrm{p}<0.05, * *$ Significant at $\mathrm{p}<0.01, * * *$ Significant at $\mathrm{p}<0.001$, NS: non significant. 
Table (3): Effect of genotype and generation on least squares means of live body weight, shank and keel length and breast width of the two genotypes studied at 8 and 12 weeks of age for males and females of the $3^{\text {rd }}$ generation

\begin{tabular}{|c|c|c|c|c|c|c|}
\hline Age & Genotype & Sex & $\begin{array}{l}\text { Live body } \\
\text { Weight }\end{array}$ & Shank length & Keel length & $\begin{array}{l}\text { Breast } \\
\text { width }\end{array}$ \\
\hline \multirow{11}{*}{$\begin{array}{l}8 \\
\text { wk }\end{array}$} & \multirow[t]{2}{*}{ 7/8SMx1/8Bj } & $\mathrm{M}$ & $585.5 \pm 21.6$ & $6.81 \pm 0.27$ & $6.13 \pm 0.30$ & $4.36 \pm 0.22$ \\
\hline & & $\mathrm{F}$ & $455.2 \pm 12.8$ & $6.83 \pm 0.14$ & $6.10 \pm 0.15$ & $4.40 \pm 0.09$ \\
\hline & \multicolumn{2}{|l|}{ Average } & $494.5 \pm 13.3$ & $6.83 \pm 0.13$ & $6.11 \pm 0.14$ & $4.39 \pm 0.09$ \\
\hline & Bj.Selected & $\mathrm{M}$ & $482.5 \pm 27.5$ & $5.70 \pm 0.30$ & $5.0 \pm 0.5$ & $3.45 \pm 0.11$ \\
\hline & & $\mathrm{F}$ & $453.9 \pm 17.14$ & $6.68 \pm 0.19$ & $6.23 \pm 0.28$ & $4.26 \pm 0.11$ \\
\hline & \multicolumn{2}{|l|}{ Average } & $456.8 \pm 15.6$ & $6.58 \pm 0.18$ & $6.11 \pm 0.27$ & $4.18 \pm 0.12$ \\
\hline & \multirow[t]{2}{*}{$\mathrm{Bj} \times \mathrm{Bj}$} & $\mathrm{M}$ & $499.0 \pm 20.2$ & $7.44 \pm 0.32$ & $6.76 \pm 0.41$ & $5.00 \pm 0.24$ \\
\hline & & $\mathrm{F}$ & $445.1 \pm 10.2$ & $6.98 \pm 0.14$ & $6.24 \pm 0.15$ & $4.55 \pm 0.08$ \\
\hline & \multicolumn{2}{|l|}{\begin{tabular}{|l} 
Average \\
\end{tabular}} & $452.4 \pm 9.5$ & $7.04 \pm 0.13$ & $6.31 \pm 0.14$ & $4.61 \pm 0.08$ \\
\hline & \multirow{2}{*}{\multicolumn{2}{|c|}{$\begin{array}{l}\text { Overall mean of males } \\
\text { Overall mean of females }\end{array}$}} & $554.5 \pm 17.0$ & $6.90 \pm 0.21$ & $6.22 \pm 0.24$ & $4.48 \pm 0.17$ \\
\hline & & & $450.5 \pm 7.3$ & $6.87 \pm 0.09$ & $6.19 \pm 0.10$ & $4.44 \pm 0.05$ \\
\hline \multicolumn{3}{|c|}{ Overall mean of 8 weeks } & $471.7 \pm 8.5^{\mathrm{b}}$ & $6.88 \pm 0.09^{\mathrm{b}}$ & $6.19 \pm 0.09^{\mathrm{b}}$ & $4.50 \pm 0.06$ \\
\hline \multirow{11}{*}{$\begin{array}{l}12 \\
\text { wk }\end{array}$} & \multirow{2}{*}{\multicolumn{2}{|c|}{ 7/8SMx1/8Bj $\mathrm{F}$}} & $937.4 \pm 29.2$ & $7.97 \pm 0.19$ & $7.58 \pm 0.22$ & $4.77 \pm 0.15$ \\
\hline & & & $783.2 \pm 18.8$ & $7.90 \pm 0.13$ & $7.43 \pm 0.11$ & $4.85 \pm 0.16$ \\
\hline & \multicolumn{2}{|c|}{ Average } & $832.1 \pm 18.2$ & $7.92 \pm 0.10$ & $7.48 \pm 0.10$ & $4.82 \pm 0.08$ \\
\hline & \multirow[t]{2}{*}{ Selected L. } & $\mathrm{M}$ & $983.5 \pm 62.5$ & $7.42 \pm 0.24$ & $7.08 \pm 0.24$ & $4.50 \pm 0.32$ \\
\hline & & $\mathrm{F}$ & $765.3 \pm 24.9$ & $7.44 \pm 0.14$ & $7.04 \pm 0.15$ & $4.90 \pm 0.15$ \\
\hline & \multicolumn{2}{|c|}{$\begin{array}{l}\text { Overall me: } \\
\text { Bj.Selected L. }\end{array}$} & 8 & $7.44 \pm 0.12$ & $7.05 \pm 0.13$ & $4.82 \pm 0.14$ \\
\hline & \multirow[t]{2}{*}{$\mathrm{Bj} \times \mathrm{Bj}$} & $\mathrm{M}$ & $880.9 \pm 128.4$ & $7.38 \pm 0.40$ & $7.13 \pm 0.52$ & $4.90 \pm 0.56$ \\
\hline & & $\mathrm{F}$ & $726.4 \pm 65.3$ & $7.08 \pm 0.17$ & $6.75 \pm 0.25$ & $4.93 \pm 0.34$ \\
\hline & \multicolumn{2}{|l|}{ Average } & $803.6 \pm 72.8$ & $7.23 \pm 0.21$ & $6.94 \pm 0.27$ & $4.91 \pm 0.30$ \\
\hline & \multirow{2}{*}{\multicolumn{2}{|c|}{$\begin{array}{l}\text { Overall mean of males } \\
\text { Overall mean of females }\end{array}$}} & $939.1 \pm 27.8$ & $7.78 \pm 0.15$ & $7.42 \pm 0.17$ & $4 . .73 \pm 0.14$ \\
\hline & & & $773.8 \pm 14.5$ & $7.70 \pm 0.09$ & $7.26 \pm 0.09$ & $4.87 \pm 0.03$ \\
\hline \multicolumn{3}{|c|}{ Overall mean of 12 weeks } & $822.4 \pm 15.0^{\mathrm{a}}$ & $7.72 \pm 0.08^{\mathrm{a}}$ & $7.30 \pm 0.08^{\mathrm{a}}$ & $483 \pm 0.07$ \\
\hline
\end{tabular}

SM: Silver Montazah, Bj: Baheij strains, Bj.Selected L.: Baheij selected line, M: Male, F: Female, - Means having different letters in every column and within every main factor of treatments are significantly different $(\mathrm{p}<0.05)$,

- Genotype had no significant effect on all traits studied except that for body weight where it affected significantly $(\mathrm{p}<0.01)$, and the interactions of Age $\mathrm{x}$ Genotype, Age $\mathrm{x}$ Sex, and Genotype $x$ Sex had no significant effects on all traits studied. 
Cont. Table (3).

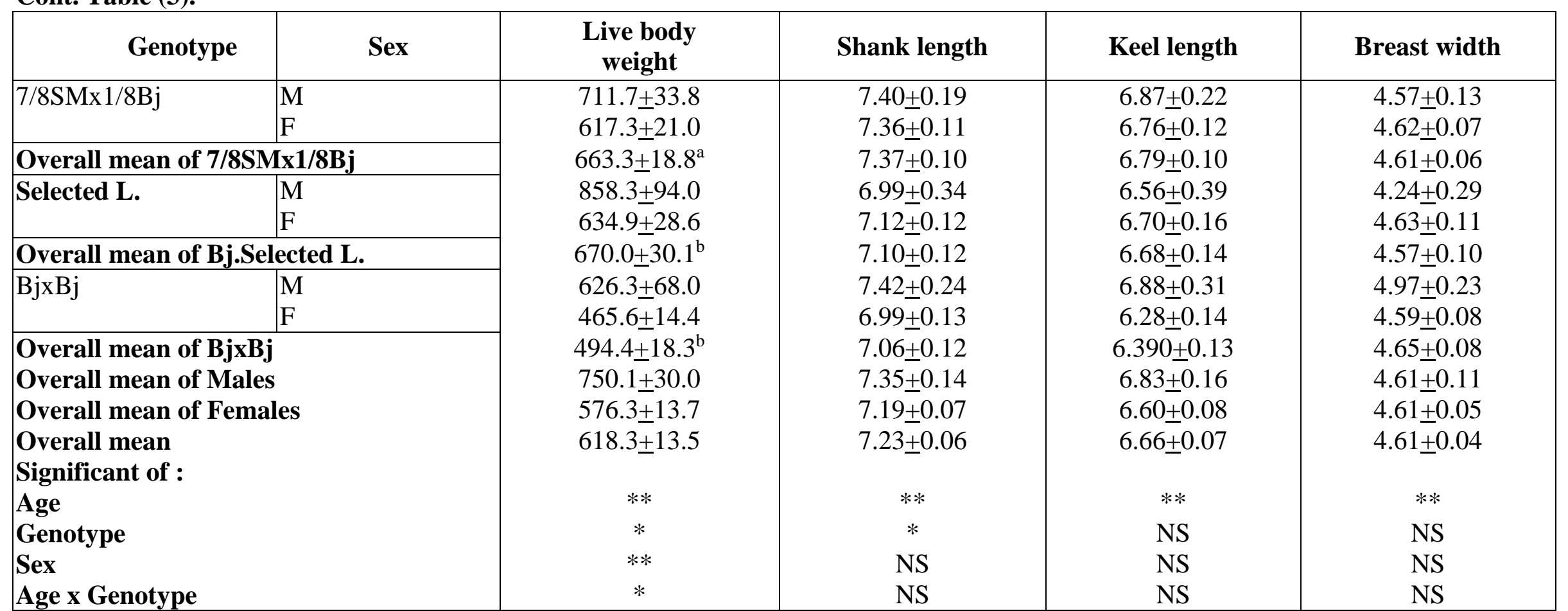

SM: Silver Montazah, Bj: Baheij strains, Bj.Selected L.: Baheij selected line, M: Male, F: Female,

- Means having different letters in every column and within every main factor of treatments are significantly different $(\mathrm{p}<0.05)$.

- Genotype had no significant effect on all traits studied except that for body weight where it affected significantly $(\mathrm{p}<0.01)$, and the interactions of Age $\mathrm{x}$ Genotype, Age x Sex, and Genotype $\mathrm{x}$ Sex had no significant effects on all traits studied.

* Significant at $\mathrm{p}<0.05, * *$ Significant at $\mathrm{p}<0.01, \mathrm{NS}$ : non significant. 
Table (4): Effect of genotype and generation on least squares means of viability of male and female birds at different periods studied

Table (4): Effect of genotype and generation on least squares means of viability of male and female birds at

\begin{tabular}{|c|c|c|c|c|c|c|}
\hline \multirow[b]{2}{*}{ Generation } & \multirow[b]{2}{*}{ Genotype } & \multicolumn{5}{|c|}{ Chicks viability (week) } \\
\hline & & $0-4$ & & 4-8 & & $0-8$ \\
\hline \multirow{4}{*}{1} & & Non sexed & Males & Females & Average & Non sexed \\
\hline & 1/2SMx $1 / 2 \mathrm{Bj}$ & $89.42+2.39$ & $98.89 \pm 1.65$ & $100.00 \pm 0.89$ & $99.44 \pm 0.63$ & $88.91+2.32$ \\
\hline & Bj.Selected L1 & $88.84+2.56$ & $100.0 \pm 0.87$ & $00.0 \pm 0.89$ & $100.0 \pm 0.62$ & $87.83+2.61$ \\
\hline & $\mathrm{Bj} \mathrm{jBj}$ & $95.56 \pm 5.35$ & $100.00 \pm 2.61$ & $100.00 \pm 1.7$ & $100.00 \pm 1.33$ & $97.15 \pm 6.03$ \\
\hline \multicolumn{2}{|l|}{ Overall mean } & $91.26 \pm 2.31^{b}$ & $99.63 \pm 0.79$ & $100 . \pm 0.72$ & $99.81 \pm 0.53^{\mathrm{a}}$ & $91.30 \pm 2.31$ \\
\hline \multirow[t]{3}{*}{2} & 3/4SMx1/4Bj & $94.07+2.32$ & $95.28 \pm 0.87$ & $97.32 \pm 0.87$ & $96.30 \pm 0.61$ & $90.20 \pm 2.61$ \\
\hline & Bj.Selected L2 & $95.87 \pm 2.63$ & $99.09 \pm 0.89$ & $96.70 \pm 0.90$ & $96.89 \pm 0.63$ & $93.82+2.69$ \\
\hline & $\mathrm{Bj} \times \mathrm{Bj}$ & $95.97 \pm 4.63$ & $98.21 \pm 1.20$ & $95.39 \pm 1.74$ & $96.80 \pm 1.23$ & $92.98 \pm 5.22$ \\
\hline \multicolumn{2}{|l|}{ Overall mean } & $95.31 \pm 2.10^{\mathrm{a}}$ & $97.53 \pm .72$ & $96.47 \pm 0.72$ & $96.99 \pm 0.50^{\mathrm{b}}$ & $92.33 \pm 2.09$ \\
\hline \multirow[t]{3}{*}{3} & 7/8SMx1/8Bj & $94.22+2.39$ & $100.00 \pm 2.85$ & $98.84 \underline{+0.89}$ & $99.42 \pm 0.63$ & $93.83 \pm 2.69$ \\
\hline & Bj.Selected L3 & $91.27 \pm 2.34$ & $100.0 \pm 0.80$ & $99.52 \pm 0.80$ & $99.76 \pm 0.57$ & $89.97 \pm 2.40$ \\
\hline & $\mathrm{Bj} \times \mathrm{Bj}$ & $97.92 \pm 4.63$ & $100.00 \pm 1.74$ & $100.00 \pm 2.01$ & $100.00 \pm 1.33$ & $95.99 \pm 5.22$ \\
\hline \multicolumn{2}{|l|}{ Overall mean } & $94.47 \pm 2.0 \mathrm{~A}^{\mathrm{b}}$ & $100.0 \pm 0.71$ & $99.45 \pm 0.78$ & $99.73 \pm 0.53^{\mathrm{a}}$ & $93.26 \pm 2.06$ \\
\hline \multicolumn{2}{|c|}{ Crossbred overall mean } & $92.57 \pm 1.37$ & $98.06 \pm 0.94$ & $98.72 \pm 0.94$ & $98.39 \pm 0.36$ & $90.98 \pm 1.50$ \\
\hline \multicolumn{2}{|c|}{ Bj.Selected line overall mean } & $91.99 \pm 1.45$ & $99.70 \pm 0.49$ & $98.74 \underline{-0.50}$ & $99.22 \pm 0.35$ & $90.54 \pm 1.44$ \\
\hline \multicolumn{2}{|c|}{ Baheij overall mean } & $96.48 \pm 2.82$ & $99.40 \pm 1.76$ & $98.46 \pm 1.94$ & $98.93 \pm 0.74$ & $95.38 \pm 3.10$ \\
\hline \multicolumn{2}{|c|}{ Genotypes overall mean } & 92.67 & $99.05 \pm 0.70$ & $98.64+0.78$ & 98.83 & 91.15 \\
\hline
\end{tabular}

- Means having different letters in every column and within every factor of treatments are significantly different ( $\mathrm{p}<0.05)$,

- All main factors studied and all interactions between them were not significant except generation which affected $(\mathrm{p}<0.01)$ viability at $4-8$ period. 
Table (5): Effect of genotype and generation on least squares means of live body weight ( $\mathrm{g}$ ), and initial weights (g) and percentages of carcass traits at 16 and 20 weeks age at the $2^{\text {nd }}$ and $3^{\text {rd }}$ generations

\begin{tabular}{|c|c|c|c|c|c|c|c|c|c|c|c|}
\hline $\begin{array}{l}\text { Gener- } \\
\text { ation }\end{array}$ & Genotype & $\begin{array}{c}\text { Age, } \\
\text { wk }\end{array}$ & Live body wt & \multicolumn{2}{|c|}{ Carcass } & \multicolumn{2}{|c|}{ Gizzard } & \multicolumn{2}{|c|}{ Liver } & \multicolumn{2}{|c|}{ Heart } \\
\hline \multirow[t]{12}{*}{ acon } & \multirow{3}{*}{ 3/4SMx1/4B } & & & Wt. & $\%$ & Wt. & $\%$ & Wt. & $\%$ & Wt. & $\%$ \\
\hline & & 16 & $1014 \pm 13$ & $711.7 \pm 22$ & $70.1 \pm 2.1$ & $38.5 \pm 2.3$ & $3.8 \pm 0.2$ & $28.2 \pm 1.5$ & $2.8 \pm 0.1$ & $8.8 \pm 0.9$ & $0.87 \pm 0.6$ \\
\hline & & 20 & $1336 \pm 15$ & $1055 \pm 24$ & $78.9 \pm 2.5$ & $43.1 \pm 2.3$ & $3.2 \pm 0.2$ & $30.8 \pm 1.6$ & $2.3 \pm 0.1$ & $10 . \overline{1} \pm 0.9$ & $0.75 \pm 0.7$ \\
\hline & \multicolumn{2}{|l|}{ Average } & $1175 \pm 10$ & $883 \pm 16$ & $74.5 \pm 1.5$ & $40.8 \pm 1.7$ & $3.5 \pm 0.2$ & $29.5 \pm 1.1$ & $2.5 \pm 0.1$ & $9.4 \pm 0.6$ & $0.81 \pm 0.4$ \\
\hline & \multirow[t]{2}{*}{ Bj.Selected L. } & 16 & $980 \pm 11$ & $718.1 \pm 21$ & $73.3 \pm 1.8$ & $32.9 \pm 1.9$ & $3.36 \pm 0.2$ & $25.25 \pm 1.2$ & $2.58 \pm 0.1$ & $7.59 \pm 0.6$ & $0.77 \pm .05$ \\
\hline & & 20 & $130 \overline{5} \pm 10$ & $982 \pm \overline{27}$ & $75.3 \pm 2.3$ & $33.6 \pm 2.5$ & $2.57 \pm 0.1$ & $29.08 \pm 1.5$ & $2.24 \pm 0.1$ & $8.18 \pm 0.7$ & $0.60 \pm .07$ \\
\hline & \multicolumn{2}{|l|}{\begin{tabular}{|l} 
Average \\
\end{tabular}} & $1143 \pm 10$ & $850 \pm 17$ & $74.3 \pm 1.4$ & $33.3 \pm 1.6$ & $2.96 \pm .13$ & $27.17 \pm 1.0$ & $2.41 \pm .08$ & $7.88 \pm 0.5$ & $0.69 \pm .04$ \\
\hline & \multirow[t]{2}{*}{ Bahej } & 16 & $945 \pm 15$ & $655 \pm 27$ & $69.3 \pm 2.3$ & $30.6 \pm 2.7$ & $3.2 \pm 0.2$ & $24.6 \pm 1.6$ & $2.6 \pm 0.1$ & $7.04 \pm 0.9$ & $0.75 \pm 0.1$ \\
\hline & & 20 & $118 \overline{1} \pm 15$ & $895 \pm 27$ & $75.8 \pm 2.3$ & $31.5 \pm 2.7$ & $2.7 \pm 0.2$ & $33.2 \pm 1.6$ & $2.8 \pm 0.1$ & $10.4 \pm 0.9$ & $0.88 \pm 0.1$ \\
\hline & \multicolumn{2}{|l|}{ Average } & $1063 \pm 11$ & $775 \pm 19$ & $72.6 \pm 1.6$ & $31.0 \pm 1.9$ & $3.0 \pm 0.2$ & $28.9 \pm 1.1$ & $2.7 \pm 0.1$ & $8.7 \pm 0.6$ & $0.82 \pm 0.1$ \\
\hline & \multirow{2}{*}{\multicolumn{2}{|c|}{$\begin{array}{l}\text { Overall mean at } 16 \mathrm{wk} \\
\text { Overall mean at } 20 \mathrm{wk}\end{array}$}} & $980+\overline{10}$ & $695+14$ & $70.9+1.2$ & $34.0 \pm 1.3$ & $3.4 \overline{7}+.1$ & $26.0+0.8$ & $2.6 \overline{5}+0.07$ & $7.8+0.4$ & $0.80+.03$ \\
\hline & & & $127 \overline{4} \pm 9$ & $977 \pm 15$ & $76.7 \pm 1.3$ & $36.1 \pm 1.4$ & $2.82 \pm 0.1$ & $31.04+0.8$ & $2.46+.07$ & $9.5 \overline{5} \pm 0.4$ & $0.75 \pm .04$ \\
\hline \multicolumn{3}{|c|}{ Overall mean of generation 2} & $1127+6$ & $836 \pm 10^{b}$ & $73.8 \pm 0.8$ & $35.0 \pm 0.9^{b}$ & $3.1 \pm 0.8$ & $29.0 \pm 0.8$ & $2.55 \pm 0.04$ & $8.68 \pm 0.3$ & $0.77 \pm 0.07$ \\
\hline \multirow{11}{*}{3} & \multirow{2}{*}{$7 / 8 \mathrm{SM} x 1 / 8 \mathrm{Bj}$} & 16 & $1348 \pm 15$ & $1109+27$ & $82.3 \pm 2.3$ & $44.1 \pm 2.5$ & $3.3 \pm 0.2$ & $27.0 \pm 1.6$ & $2.0 \pm 0.1$ & $9.6 \pm 0.9$ & $0.71 \pm 0.1$ \\
\hline & & 20 & $1102 \pm 12$ & $1302 \pm 25$ & $81.3 \pm 1.3$ & $42.0 \pm 2.1$ & $2.6 \pm 0.2$ & $26.6 \pm 1.4$ & $1.7 \pm 0.1$ & $15 . \overline{9} \pm 0.7$ & $0.99 \pm 0.05$ \\
\hline & \multicolumn{2}{|l|}{ Average } & $1475 \pm 10$ & $1206 \pm 1$ & $81.8 \pm 1.1$ & $43.0 \pm 1.9$ & $2.9 \pm 0.2$ & $26.8 \pm 1.1$ & $1.8 \pm 0.1$ & $12.7 \pm 0.6$ & $0.85 \pm 0.5$ \\
\hline & \multirow{2}{*}{ Bj.Selected L. } & 16 & $1294 . \overline{8} \pm 15$ & $1012 \pm 27$ & $78.1 \pm 2.2$ & $39.8 \pm 2.5$ & $3.0 \overline{6} \pm 0.2$ & $25.1 \overline{4} \pm 1.5$ & $1.9 \overline{4} \pm 0.1$ & $7.70 \pm 0.8$ & $0.59 \pm .07$ \\
\hline & & 20 & $1584 \pm 15$ & $1292 \pm 27$ & $81.5 \pm 2.2$ & $40.6 \pm 2.5$ & $2.56 \pm 0.2$ & $25.80 \pm 1.5$ & $2.63 \pm 0.1$ & $15.8 \pm 0.8$ & $1.0 \pm .07$ \\
\hline & \multicolumn{2}{|l|}{ Average } & $1439.8 \pm 11$ & $1152 \pm .19$ & $79.8 \pm 1.4$ & $40.1 \pm 1.7$ & $2.81 \pm .14$ & $25.47 \pm 1.0$ & $1.79 \pm .59$ & $11.7 \overline{5} \pm 0.5$ & $0.80 \pm .04$ \\
\hline & Bahej & 16 & $974 \pm 15$ & $670 \pm \overline{2} 7$ & $68.8 \pm 2.3$ & $32.0 \pm 2.5$ & $3.3 \pm 0.2$ & $22.1 \pm \overline{1} .6$ & $2.3 \pm \overline{0} .1$ & $7.1 \pm 0.9$ & $0.73 \pm 0.07$ \\
\hline & & 20 & $118 \overline{6} \pm 15$ & $882 \pm 27$ & $74.4 \pm 2.2$ & $42.4 \pm 2.6$ & $3.6 \pm 0.2$ & $36.0 \pm 1.6$ & $3.0 \pm 0.1$ & $12 . \overline{2} \pm 0.9$ & $1.03 \pm 0.7$ \\
\hline & \begin{tabular}{|l} 
Average \\
\end{tabular} & & $1080 \pm 11$ & $776 \pm 17$ & $71.6 \pm 1.6$ & $37.2 \pm 1.8$ & $3.4 \pm 1.2$ & $29.1 \pm 1.1$ & $2.7 \pm 0.1$ & $9.7 \pm 0.6$ & $0.88 \pm 0.4$ \\
\hline & Overall mean at & $6 \mathrm{wk}$ & $1206 \pm 8.0$ & $930 \pm 15$ & $76.4 \pm 1.2$ & $38.6 \pm 1.4$ & $3.21 \pm 0.11$ & $24.77 \pm 0.8$ & $2.07 \pm .07$ & $8.13 \pm 0.5$ & $0.68 \pm .04$ \\
\hline & Overall mean at 2 & $0 \mathrm{wk}$ & $1458 \pm 8.0$ & $1159 \pm 15$ & $79.1 \pm 1.2$ & $41.7 \pm 1.4$ & $2.92 \pm 0.11$ & $29.46 \pm 0.8$ & $2.11 \pm .06$ & $14.63 \pm 0.5$ & $1.00 \pm .03$ \\
\hline \multicolumn{3}{|c|}{ Overall mean of generation 3} & $1331.6 \pm 6.0^{\mathrm{a}}$ & $1044.6 \pm 11^{\mathrm{a}}$ & $77.8 \pm 0.9$ & $40.14 \pm 1.0^{\mathrm{a}}$ & $3.06 \pm 0.08$ & $27.11 \pm 0.6$ & $2.09+.05$ & $11.38 \pm 0.3$ & $0.84+.02$ \\
\hline
\end{tabular}


Cont. Table (5).

\begin{tabular}{|c|c|c|c|c|c|c|c|c|c|c|}
\hline Gener- & Genotype & Live body & Cal & cass & Giz & zard & $\mathbf{L i}$ & & $\mathbf{H e}$ & \\
\hline & & & Wt. & $\%$ & Wt. & $\%$ & Wt. & $\%$ & Wt. & $\%$ \\
\hline Overal & I mean of 16 wks of age & $1092.7 \pm 5.9^{y}$ & $812.6 \pm 10^{y}$ & $73.7 \pm 0.9$ & $36.31 \pm 1.0^{y}$ & $3.34 \pm .08$ & $25.39 \pm 0.6^{\mathrm{y}}$ & $2.36 \pm .04$ & $7.97 \pm 0.3^{y}$ & $0.74 \pm .02$ \\
\hline Overal & 1 mean of 20 wks of age & $1365.8 \pm 6.0^{x}$ & $1068+11^{x}$ & $77.9 \pm 0.9$ & $38.9 \pm 1.0^{x}$ & $2.87 \pm .08$ & $30.29 \pm 0.6^{\mathrm{X}}$ & $2.28 \pm .05$ & $12.09 \pm 0.5^{\mathrm{x}}$ & $0.88 \overline{ \pm} .02$ \\
\hline $\begin{array}{l}\text { Overal } \\
\text { group }\end{array}$ & I mean of upgrading & $1325 \pm^{\mathrm{a}}$ & $1045 \pm 11^{\mathrm{a}}$ & $78.2 \pm 0.8$ & $41.9 \pm 1.3^{\mathrm{a}}$ & $3.2 \pm 0.10$ & $28.1 \pm 0.75^{\mathrm{b}}$ & $2.2 \pm 0.10$ & $11.10 \pm 0.4^{\mathrm{a}}$ & $0.83 \pm 0.0$ \\
\hline $\begin{array}{l}\text { Overal } \\
\text { line }\end{array}$ & I mean of Bj.Selected & $1291.2 \pm 7.2^{b}$ & $1001 \pm 1.3^{b}$ & $77.1 \pm 1.1$ & $36.70 \pm 1.2^{b}$ & $2.89 \pm .09$ & $26.31 \pm .7^{c}$ & $2.10 \pm .06$ & $9.82 \pm 0.4^{b}$ & $0.83 \pm .03$ \\
\hline Overal & I mean of Bahej strain & $1072 \pm 7^{c}$ & $775 \pm 12^{c}$ & $72.1 \pm 0.8$ & $34.1 \pm 1.3^{\mathrm{b}}$ & $3.2 \pm 0.10$ & $29.1 \pm 0.8^{\mathrm{a}}$ & $2.7 \pm 0.10$ & $9.20 \pm 0.5^{b}$ & $0.84 \pm 0.0$ \\
\hline $\begin{array}{l}\text { Overal } \\
\text { Signific }\end{array}$ & $\begin{array}{l}I \text { mean } \\
\text { cance of: }\end{array}$ & 1226 & 938 & 75.74 & 37.52 & 3.11 & 27.67 & 2.32 & 10.07 & 0.81 \\
\hline Genera & ation (Gen) & $* *$ & $* *$ & 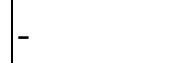 & $* *$ & 1 & NS & - & $* *$ & 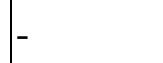 \\
\hline Genoty & pe (Get) & $* *$ & $* *$ & NS & $* *$ & NS & $* *$ & NS & $* *$ & NS \\
\hline Age & & $* *$ & $* *$ & NS & $*$ & NS & $* *$ & NS & $* *$ & NS \\
\hline
\end{tabular}

- Means having different letters in every column and within every main factor of treatments are significantly different $(\mathrm{p}<0.05)$,

- The interaction between the different main factors for most of the studied traits were not significant at $(\mathrm{p}<0.05)$,

-* Significant at $\mathrm{p}<0.05, * *$ Significant at $\mathrm{p}<0.01$, NS: non significant. 


\section{REFERENCES}

Abou-El-Ella, N., 1982. A comparative study on the performance potentiality of four locally developed strains and their $F_{1}$ crosses. M.Sc. Thesis, Fac. of Agric., Univ. of Alexandria, Egypt.

Adedeji, T. A.; Adebambo, O. A.; Peters, S. O.; Ojedapo, L. O.; and Ige, A. O., 2006. Growth performance of crossbred and purebred chickens resulting from different sire strain in a humid tropical environment. J. Anim. Vet. Adv., 5(8): 674-678.

Aly, O. M.; Abou El-Ghar, R. S.; Abou El-Ella, N. Y.; and Aly, W. Z., 2005. Using potency ratio to interpret hybrid vigor in crossing between two local strains. Egypt. Poult. Sci., 25(2): 413428.

Aly, O. M.; Abd-El-Hamid, E. A.; and Aly, W. Z., 2006. Effect of crossing on the performance of local strains. 4. Blood Hematology and Biochemical Traits and Some Organs Relative Weights of Chicken Cocks. J. Agric. Env. Sci. Alex. Univ. Egypt., 5(1): 5771

Amin, E. M., 2007. Effect of crossing on growth performance and viability of commercial and native Egyptian chicken breeds. Egypt. Poult. Sci., 27(4): 11511173.

Amin, E. M., 2008. Effect of crossing among some local and foreign strains of chicken breeds on carcass traits. Mansoura Univ., 33(3): 1063-1078.

Amin, E. M., 2009. Effect of crossing between the local Black Baladi (Bronze) and White Nicholas turkeys on productive and reproductive traits. 2 . Effect of repeated backcrossing for two generations on growth traits. Egypt. Poult. Sci., 29(3): 851-885.

Balat, Magda M.; Afify, Yousria K.; Abou El-Ella, Nazla Y.; and Breakaa, Mervat A., 2005. Breed differences for adaptation to economical feeding system. 1. Growth traits. Anim. Prod.
Res. Inst. Conf. and the Regional Symposium on Buffalo Prod. And Future Prespect., Sakha, 27-29 September 2005: 481-492.

Barbato, G.F., 1991. Genetic architecture of growth curve parameters in chickens. Theor. Appl. Genet. 83(1):24-32.

Cloete, S.W.; Bunter, K.L.; Lambrechts H.; Brand, Z.; Swart, D. and Greyling, J.P., 2006 .Variance components for live weight, body measurements and reproductive traits of pair-mated ostrich females. $\mathrm{Br}$ Poult. Sci. 47(2):147-158.

Duncan, D.B., 1955 .Multiple range and multiple F. test. Biometrics 11: 1-42.

Eitan, Y. and Soller, M. ,1995 . Two-way selection for threshold body weight at first egg in broiler strain females. 5. Replication of results in a twogeneration selection experiment. Poult. Sci.74(10):1561-1565.

El-Turkey, A.I., 1981. Hybrid vigor potence ratio in performance of crossbred from four local breeds of chickens. M.Sc. Thesis, Fac. of Agric., Alex. Univ., Egypt.

Fairfull, R.W., 1990. Heterosis, cited by "Poultry Breeding and Genetics", page 913-933. El-Sevier science publishers, B.V. New York, USA.

Gaya, L.G.; $\quad$ Ferraz, J.B.; Rezende,F.M.; Mourão,G.B. ; Mattos, E.C.; Eler,J.P. and Michelan Filho,T., 2006. Heritability and genetic correlation estimates for performance and carcass and body composition traits in a male broiler line. Poult. Sci. 85(5): 837-843.

Gaya, L.G.; Costa,A.; Ferraz, J.B.S.; Rezende,F.M.; Mattos,E.C.; Eler, J.P.; Filho,T.M.; Mourao,G.B. and Figueiredo, L.G.G., 2007. Genetic trends of absolute and relative heart weight in amale broiler line. Genet. Mol. Res. 6:1091-1096.

Gondwe, T.N. and Wollny, C.B.A., 2005. Evaluation of the Growth Potential of 
Local Chickens in Malawi. International Journal of Poult. Sci. 4 (2): 64-70.

Isguzar, E., 2003. Growth, carcass traits and meat quality of Bronze and White turkeys in Isparta province of Turkey. Arch. Tierz. 46: 473-481.

Jackson, S. and Diamond, J., 1996. Metabolic and digestive responses to artificial selection in chickens. Evolution 50:1638-1650.

Konarzewski, M.; Gavin, A.; McDevitt, R. and Wallis, I.R., 2000. Metabolic and organ mass responses to selection for high growth rates in the domestic chicken (Gallus domesticus). Physiol. Biochem. Zool.73(2):237-248.

Ledur, M.C.; Schmidt, G.S.; Figueiredo, E.A.P.;Avila,V,S.and Balen,L., 1993.

Genetic and phenotypic parameters for productive traits in White egg layer stocks. Pesqui.Agropecu.Bras. 28:10311037.

Lerner, I.H. and Asmundson V.S., 1932. Inheritance of rate of growth in domestic fowl. Sci. Agric. 12: 652.

Mahmoud,T.H.; Sayed,I.F. and Madkour, Y.H., 1974."The Silver Montazah" a new variety of chickens. Agric. Res. Rev. Vol. 52 (6): 97-105.

Mahmoud, T.H.; El-Turky, A.I.; Madkour, Y.H. and Heider,A., 1979. "Baheij" a new breed of chickens. Agric. Res. Rev., Cairo, 67:227.

Mandour, M.A.; Sharaf, M.M.; Kosba, M.A. and El-Naggar, N.M., 1992. Estimation of combining ability and heterosis for some economic traits in local and commercial broiler strains of chickens from a full dialel cross. Egypt. Poult. Sci. 12: 57-78.

Mandour M.A.; Abd-Allah,G.A. and Sharaf, M.M., 1996. Effect of crossbreeding on some carcass traits of native and standard breeds of chickens. Egypt. Poult. Sci. Vol. 16 (I): 171-185.

Mohamed, A.A., 2003. Effect of diallel crosses on poultry performance. M.Sc. Thesis, Facul. of Agric., Alex. Univ., Egypt.
Mostafa, M.Y. and Nofal, R.Y., 2000. Effect of crossing two breeds of turkey on live body measurements, growth performance and livability. Egypt. Poult. Sci. 20: 239-252.

Mulder, H.A.; Hill, W.G.; Vereijken, A. and Veerkamp,R.F., 2009. Estimation of genetic variation in residual variance in female and male broiler chickens. Animal 3(12):1673-80.

Nawar, M.E. and Abdou,F.H., 1999. Analysis of heterotic gene action and maternal effects in crossbred Fayoumi chickens. Egypt Poult. Sci. 19 (3): 671689.

Nawar, M.E.; Aly, O.M. and Abd ElHamid, A.E., 2004. The effect of crossing on some economic traits in chicken. Egypt. Poult. Sci. 24 (1): 163176.

Nestor,K.E.; Anderson, J.W.; Patterson, R.A. and Welleman, S.G., 2006. Genetics of growth and reproduction in the turkey. 16. Effect of repeated backcrossing of an Egg line to a commercial sire line. Poult. Sci. 85: 1550-1554.

Nestor,K.E.; Anderson,J.W.; Patterson, R.A. and Velleman,S.G., 2008. Genetics of growth and reproduction in the Turkey. 17. Changes in genetic parameters over forty generations of selection for increased sixteen-week body weight. Poult Sci. 87(10):19711979.

Nigussie Dana; vander Waaij, E.H.; Johan, A.M.and van Arendonk, 2011. Genetic and phenotypic parameter estimates for body weights and egg production in Horro chicken of Ethiopia. Trop. Anim. Health. Prod. 43:21-28.

Norris, D. and Ngambi,J.W., 2006. Genetic parameter estimates for body weight in local Venda chickens. Trop. Anim. Health Prod. Oct-Nov., 38(78):605-9.

Ramkrishna, R.S.; Sheriff, F.R. and Raj Manohar, G., 2012. Growth performance and carcass yields as 
influenced by age and sex in different turkey varieties. Tamilnadu J. Veterinary \& Animal Sciences 8 (2): 94100.

Rance, K. A.; McEntee,G.M. and McDevitt, R.M., 2002 .Genetic and phenotypic relationships between and within support and demand tissues in a single line of broiler chicken. Br. Poult. Sci. 43:518-527.

Rekaya, R.; Sapp,R.L.; Wing,T. and Aggrey, S.E. 2013. Genetic evaluation for growth, body composition, feed efficiency, and leg soundness. Poult. Sci. 92(4):923-929.

Sandercock, D.A.; Nute, G.R. and Hocking, P.M., 2009. Quantifying the effects of genetic selection and genetic variation for body size, carcass composition, and meat quality in the domestic fowl (Gallus domesticus) Poult. Sci. 88: 923-931.

SAS Institute, 1988. User's Guide Statistics. SAS Institute INC, Cary, NC, USA.

Scrivener, D., 2002 .Starting with Bantams. Poultry Book review, Broad Leys Publishing Ltd-website.

Sharaf, M.M.; Mandour, M.A. and Taha, A.E., 2006. Effect of dialed crossing on some growth performance, carcass traits and immune response against New Castle disease virus vaccine of Japanese quails. Egypt. Poult. Sci. 26 (4): 1451-1470.

Shebl, M.K.A.; Magda M. Balat and Nadia A. El-Sayed, 1995. Introducing Naked-Neck gene to Alexandria strain in comparison to their local crossbreds in certain economic traits. Egypt. Poult. Sci. 15: 1-5.

Williams, S.M.; Price, S.E.and Siegel, P.B. 2002. Heterosis of growth and reproductive traits in fowl. Poult. Sci. 81(8):1109-1112.

Wolc, A.; White,I.M.; Avendano,S. and Hill, W.G. 2009. Genetic variability in residual variation of body weight and conformation scores in broiler chickens. Poult. Sci. 88(6):1156-1161.

Yakubu, A. ; Idahor,K. O. and Agade, Ya'u Isopa, 2009. Using factor scores in multiple linear regression model for predicting the carcass weight of broiler chickens using body measurements. Revista Científica UDO Agrícola, Vol. 9, No. 4, October -December, 2009, pp. 963-967

Yalcin, S.; Zhang, X.; McDaniel, G.R. and Kuhlers, D.L., 2000. Effects of divergent selection for incidence of tibial dyschon droplasia (TD) on purebred and crossbred performance. 2 . Processing Yield. Br. Poult. Sci. 41(5):566-569. 
تحسين الصفات الإنتاجية لاجاج بهيج من خلال التزاوج العربى الرجعى والإنتخاب لوزن الجسم عند 8

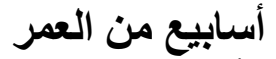

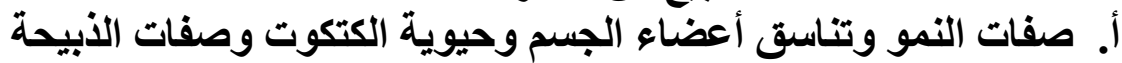

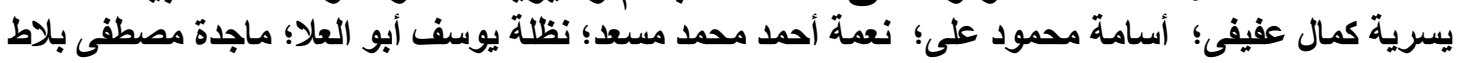

$$
\text { معهد بحوث الإنتاج الحيوانى ـ مركز البحوث الزر اعية ـ وزارة الزراعة ـ الدقى }
$$

تم إجر اء مقارنة بين ثلاث تر اكيب وراثية من نوع بهيج وهى بهيج (مجمو عة مقارنة)، خط بهيج منتخب لزيادة وزن

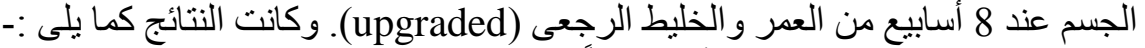

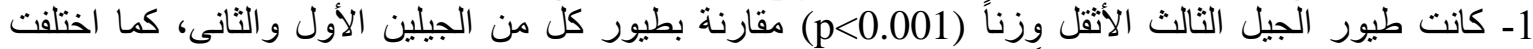

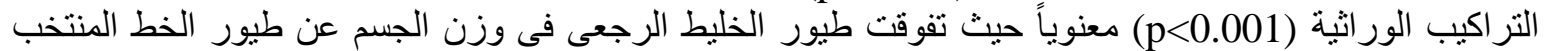

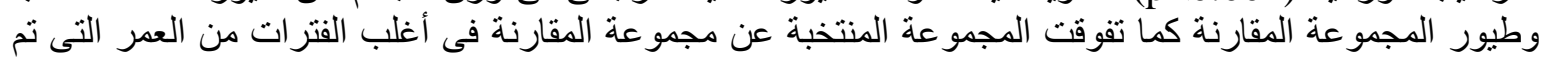
در استها.

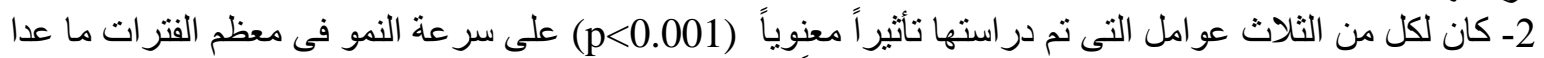
البعض منها حيث كانت طيور الجيل الثالث الأسرع نمواً (

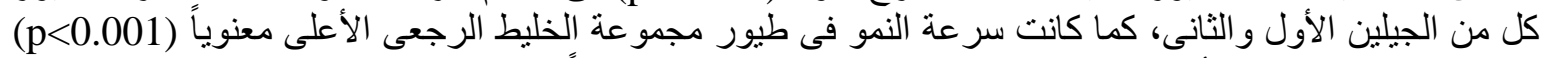

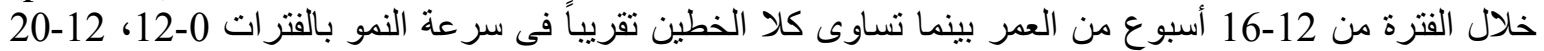

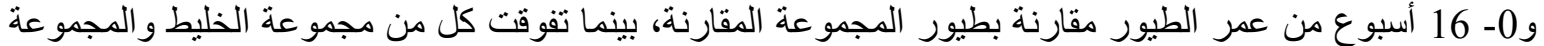

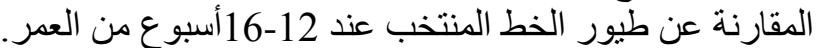

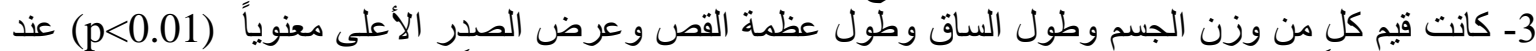

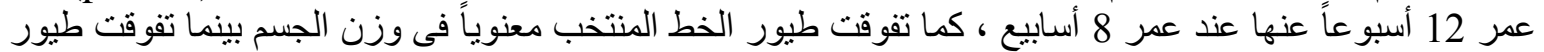
مجموعة الخليط فى طول الساق. كذللك كانت الطيور الذكور الأعلى (

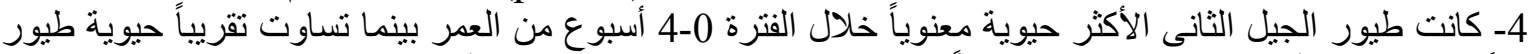

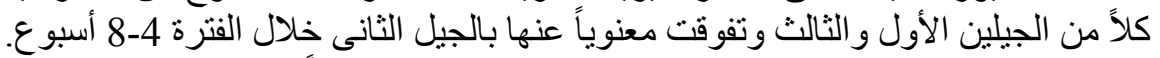

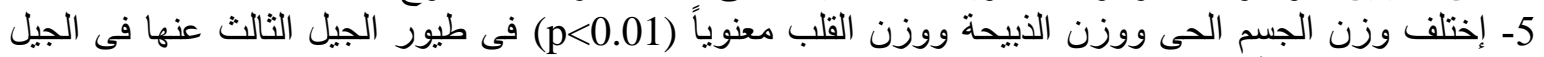

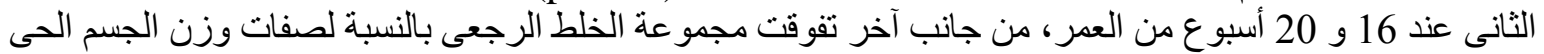

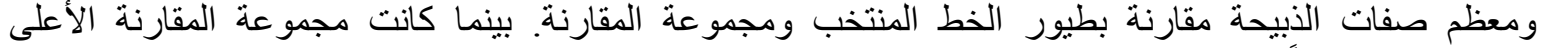
(p<0.01)

يمكن القول بأن خط الخليط الرجعى (الناتج عن خلط ذكور منتخبة عند الوزن الناضج من سلالة المنتزه الفضى بإنان الناث

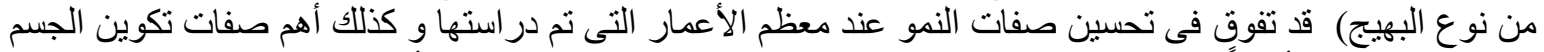

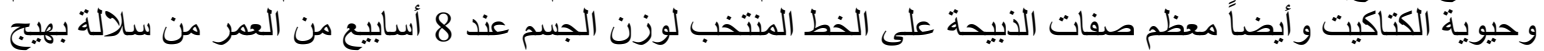
لثناث أجيال، وكذلك على طيور المجمو عة المقارنة. 